\title{
Role of frontal cortex in inferential reasoning: Evidence from the Word Context Test
}

\author{
KATRINA KEIL, ${ }^{1}$ JULIANA BALDO,${ }^{2}$ EDITH KAPLAN, ${ }^{3}$ JOEL KRAMER,${ }^{4}$ AND DEAN C. DELIS ${ }^{5}$ \\ ${ }^{1}$ Casa Colina Centers for Rehabilitation, Pomona, California \\ ${ }^{2}$ VA Northern California Health Care System, Sacramento, California \\ ${ }^{3}$ Boston University School of Medicine and Suffolk University, Boston, Massachusetts \\ ${ }^{4}$ University of California, San Francisco, California \\ ${ }^{5}$ VA Medical Center, San Diego and the University of California, San Diego, Medical School, California \\ (Received August 12, 2004; Revised December 17, 2004; Accepted March 14, 2005)
}

\begin{abstract}
Problem: Inferential reasoning in language involves the ability to deduce information based on context and prior experience. This ability has been generally studied as a right-hemisphere function. Recent research, however, has suggested that inferencing involves anterior regions of both the left and right hemispheres. Methods: We further explored this idea by testing a group of non-aphasic, focal frontal patients (right and left hemisphere) on a new test of inferencing, the Word Context Test. The Word Context Test requires examinees to identify the meaning of a made-up word (e.g., prifa) based on its use in a series of sentences. Findings: Patients with frontal lobe lesions were significantly impaired on this task relative to a group of age- and education-matched controls. Contrary to earlier research focusing on a special role for the right hemisphere in inferencing, there was considerable overlap in performance of right- and left-frontal patients, with right-frontal patients performing better. Conclusions: These findings suggest that inferencing is disrupted following focal frontal injury and have implications for discourse comprehension in non-aphasic patients. (JINS, 2005, 11, 426-433.)
\end{abstract}

Keywords: Frontal lobes, Inferencing, Reasoning, Prefrontal cortex, Cognitive control, Executive function

\section{INTRODUCTION}

Inferencing refers to the ability to make a judgment on the basis of context and prior information rather than via direct observation. Traditionally, this ability has been associated with the right hemisphere (Brownell et al., 1986; Myers \& Brookshire, 1996; Wapner et al., 1981; but see Harden et al., 1995 and Lehman-Blake \& Tompkins, 2001). The types of errors that right hemisphere patients make on inferencing tasks indicate deficits in the ability to abstract, integrate information across time, and update knowledge based on new information (Brownell et al., 1986; Hier \& Kaplan, 1980; Myers \& Brookshire, 1996). More recent work has suggested that inferential processes rely on anterior brain regions in both the right and left hemisphere (Ferstl et al., 2002; Ferstl \& von Cramon, 2001; McDonald, 1993;

Reprint requests to: Dr. Katrina Keil, Transitional Living Center, Casa Colina Centers for Rehabilitation, 255 East Bonita Ave., Pomona, CA 91769-6001. E-mail: trina.keil@ aya.yale.edu
McDonald \& Pearce, 1996). This work is consistent with deficits of integration and updating that have long been reported in patients with frontal injury on tests of reasoning and problem solving (Baldo et al., 2004; Lezak, 1995; Milner $\&$ Petrides, 1984).

What has been less well-studied is whether such reasoning deficits in non-aphasic frontal patients also result in impairment on language-based inferencing tasks. Novoa and Ardila (1987) tested a group of non-aphasic prefrontal patients on a battery of language tests and reported significant language difficulties, especially in left-prefrontal patients, that included perseveration, reduced initiation, concreteness, and confabulation. Similar results have also been reported by Alexander et al. (1989), Kaczmarek (1984), and Pearce et al. (1998). Pearce et al. tested a small group of patients with focal frontal pathology (due to traumatic brain injury) on their ability to generate both literal and inferred meanings of ambiguous product advertisements (e.g., for a deodorant, "Fascinating things happen on Impulse"). While these patients were generally able to pro- 
duce competent interpretations of the literal meanings of the advertisements, they were disproportionately impaired in their ability to generate non-literal interpretations. That is, they performed in a concrete manner and were less able to generate abstract interpretations.

A more recent study tested frontal and non-frontal patients on an inferencing task that involved deciding whether sentence pairs were pragmatically coherent (e.g., "Mary's exam was about to start. Therefore, her palms were sweaty"; Ferstl et al., 2002). The study found that patients with left- and bilateral-frontal (but not right-frontal) lesions were significantly impaired on this task. That is, these patients were unable to recognize the implied connection between the sentence pairs. The same task was also used in an fMRI study with normal participants and was found to activate left dorsomedial prefrontal cortex (as well as a number of other regions) in a comparison of coherent versus incoherent sentence pairs (Ferstl \& von Cramon, 2001). Ferstl and colleagues concluded that lateral prefrontal cortex is involved when discourse comprehension requires "nonautomatic, selfguided cognitive processes" (p. 306).

It is possible that such inferencing deficits in frontal lobe patients are due to more basic impairments in cognitive processes such as working memory and word retrieval. Working memory has been shown to be disrupted in this patient group (e.g., Baldo \& Shimamura, 2000; Freedman \& OscarBerman, 1986) and could affect patients' ability to hold multiple arguments in mind, while at the same time attempting to abstract some relationship between them. Also, basic deficits in language use such as word retrieval have been associated with impaired performance on inferencing tasks (Ferstl et al., 2002). Word retrieval, as measured by verbal fluency tasks, has been shown to be impaired in patients with frontal lobe lesions (e.g., Baldo \& Shimamura, 1998; Henry \& Crawford, 2004; Troyer et al., 1998).

The goal of the current study was to further examine the role of prefrontal cortex in inferential reasoning. To do this, we tested non-aphasic, focal frontal patients' ability to infer and integrate relevant information across a series of sentences. The task used was a new measure, the Word Context Test (Delis et al., 2001), which requires participants to deduce the meaning of a made-up word (e.g., prifa) based on its use in a series of sentences (e.g., "You need to prifa to grow"). This task was originally designed to study language acquisition in children and was shown to be sensitive to deficits in cognitive flexibility and the ability to maintain set (Werner \& Kaplan, 1950). The current study was the first to test focal frontal patients on this new measure, and it represents both a preliminary validation study as well as an examination of the cognitive processes underlying performance on the test. The patients in this study were not part of the test development but were tested on the final version of the Word Context Test prior to publication. It was predicted that frontal patients would be impaired on this task relative to controls. In order to understand the source of patients' deficits, we performed more qualitative analyses to test whether inferencing deficits arose from a failure to integrate information either within or across sentences, and/or a failure to maintain set. Based on previous work with this patient group, it was predicted that inferencing deficits would arise as a result of frontal patients' inability to integrate information across sentences. Last, we compared performance on the Word Context Test to performance on measures of supraspan memory and verbal fluency, in order to explore the role that working memory and word retrieval, respectively, might play in performance on this task.

\section{METHODS}

\section{Research Participants}

Twelve patients with prefrontal cortex lesions ( 8 male and 4 female) and twelve age- and education-matched controls from the same community ( 8 male and 4 female) participated in the study (see Table 1 for participant characterization). The participants ranged in age from 54-81 years except for one 35 year-old patient and his age-matched control. There was no difference in age between patients and controls $[F(1,22)=.08, n s ; M=65.08$ and 63.67 years, respectively], or education $[F(1,22)=.84, n s ; M=14.08$ and 14.92 years, respectively]. Eleven participants in each group were right-handed; 1 patient and 1 control were left-handed.

Lesion etiologies included stroke $(n=7)$, surgery for meningioma $(n=2)$, aneurysm $(n=1)$, cyst $(n=1)$, and arterio-venous malformation $(n=1)$. Lesions were localized in lateral prefrontal cortex in ten patients and in ventral frontal cortex in 2 patients ( 5 and 6 ). Seven patients had left-hemisphere lesions and the remaining 5 had righthemisphere lesions (see Figure 1 for lesion reconstructions). Patients were excluded if they had lesions that extended beyond frontal cortex based on MRI and CT review. The average number of years post onset of injury was 10 years (range $=1-18$ years). Exclusion criteria for all participants included prior history of drug or alcohol abuse, psychiatric disorders, or neurologic disorders. Patients were additionally screened for aphasia; all scored within normal limits on the Western Aphasia Battery (Kertesz, 1982).

Testing was completed at the VA Northern California Health Care System in Martinez, CA. The study was approved by the Institutional Review Board, and all participants read and signed consent forms prior to participation.

\section{Materials}

The Word Context Test was administered as part of a larger executive functions battery (Delis-Kaplan Executive Functions System; Delis et al., 2001). It consisted of one practice trial and 10 target trials. On each trial, there was a series of five sentences that were presented in black print on a single page in a stimulus booklet. The examiner used a sheet of white paper to cover sentences so that only one sentence was revealed at a time. The first sentence provided very little specific information as to the identity of the cor- 
Table 1. Participant characterization

\begin{tabular}{|c|c|c|c|c|c|c|c|c|}
\hline Patient & Gender & $\begin{array}{l}\text { Lesion } \\
\text { site }\end{array}$ & $\begin{array}{l}\text { Volume } \\
\text { (cc) }\end{array}$ & $\begin{array}{l}\text { Lesion } \\
\text { etiology }\end{array}$ & $\begin{array}{l}\text { Age } \\
\text { at test }\end{array}$ & $\begin{array}{l}\text { Years } \\
\text { post }\end{array}$ & $\begin{array}{l}\text { Education } \\
\text { (years) }\end{array}$ & $\begin{array}{c}\text { Total } \\
\text { guesses }\end{array}$ \\
\hline 1 & M & $\mathrm{L}$ & 17.5 & Stroke & 66 & 14 & 14 & 36 \\
\hline 2 & $\mathrm{~F}$ & $\mathrm{~L}$ & 26.2 & Stroke & 76 & 12 & 14 & 46 \\
\hline 3 & $\mathrm{~F}$ & $\mathrm{~L}$ & 27.9 & Meningioma & 70 & 18 & 16 & 47 \\
\hline 4 & M & $\mathrm{L}$ & 41.1 & Stroke & 69 & 2 & 15 & 55 \\
\hline 5 & M & $\mathrm{L}$ & 18.8 & Stroke & 54 & 1 & 11 & 41 \\
\hline 6 & M & $\mathrm{L}$ & - & Meningioma & 64 & 11 & 16 & 37 \\
\hline 7 & M & $\mathrm{L}$ & - & Stroke & 67 & 5 & 13 & 49 \\
\hline 8 & $\mathrm{~F}$ & $\mathrm{R}$ & 17.3 & Stroke & 81 & 16 & 12 & 32 \\
\hline 9 & $\mathrm{~F}$ & $\mathrm{R}$ & 12.9 & Stroke & 78 & 3 & 12 & 50 \\
\hline 10 & M & $\mathrm{R}$ & 25.9 & Cyst & 54 & 10 & 18 & 33 \\
\hline 11 & M & $\mathrm{R}$ & 24.5 & AVM & 35 & 14 & 12 & 47 \\
\hline 12 & M & $\mathrm{R}$ & 200.4 & Aneurysm & 67 & 18 & 17 & 29 \\
\hline All frontal & $8 \mathrm{M}, 4 \mathrm{~F}$ & $7 \mathrm{~L}, 5 \mathrm{R}$ & $41.3 \pm 56.5^{*}$ & - & $65.1 \pm 12.6^{*}$ & $10.0 \pm 6.2^{*}$ & $14.1 \pm 2.2 *$ & $41.8 \pm 8.3^{*}$ \\
\hline All control & $8 \mathrm{M}, 4 \mathrm{~F}$ & - & - & - & $63.7 \pm 11.2 *$ & - & $14.9 \pm 2.2 *$ & $32.9 \pm 5.7 *$ \\
\hline
\end{tabular}

Note. Dashes indicate data that are not available or not applicable. $\mathrm{L}=$ left hemisphere; $\mathrm{R}=$ right hemisphere; volume $=$ estimated lesion size; years post $=$ number of years post stroke; AVM = arteriovenous malformation; total guesses $=$ total number of guesses made on the Word Context Test. $* M \pm S D$.

rect response, and the subsequent sentences provided increasingly more specific contexts. The fifth and final sentence provided a fairly concrete context and made the identity of the correct response obvious to normal participants (see Table 2 for an example). The battery also included a verbal fluency task and the California Verbal Learning Test-II (CVLT-II; Delis et al., 2000). These tests were included in the current analysis because they provide conditions that assess retrieval from semantic knowledge and working memory, respectively. The CVLT-II is a list-learning task made up of 16 words, which are evenly distributed among four semantic categories ( furniture, animals, ways of traveling, and vegetables). The verbal fluency task consisted of animal naming.

\section{Procedure}

Participants were tested individually in a quiet testing room. Participants were first given instructions, which included the following: "Let's pretend you're in another country where they speak a different language. I have some words from this language, and I want you to figure out what the words mean." Participants were told that they would see and hear a series of sentences with a made-up word and that they should guess what the word might mean after every sentence. The sentences were read out loud by the examiner and also appeared in the stimulus booklet. The examiner wrote down the participant's response after every sentence. Participants were never given feedback as to whether their responses were correct or not. They were simply told to continue giving the same answer if they thought they had the correct response. A practice trial was administered first, followed by 10 target trials. Each trial consisted of five sentences, which were revealed to the participant one at a time. Participants were able to see preceding sentences within a trial, so that they could refer back to them if necessary. A sample trial and responses are given in Table 2 .

The CVLT-II and verbal fluency tasks were administered during the same testing session according to standardized instructions. The CVLT-II required participants to recall, in any order, a list of 16 words that were read by the examiner. This procedure was repeated across five trials. On the verbal fluency task, participants were asked to name as many items as possible in $90 \mathrm{~s}$ that belonged to the semantic condition.

\section{Data Analysis}

Both quantitative and qualitative analyses were done in an attempt to characterize performance on the Word Context Test. As a measure of overall performance, we calculated accuracy and the number of guesses needed to arrive at the correct response. Accuracy was calculated as the number of trials (out of 10) for which the participant provided the correct response by the final sentence of the trial. Total guesses were calculated as the number of guesses (out of 50) the participant made before arriving at and maintaining the correct response. Since the sentences within a trial become more and more concrete, this latter measure was used to determine whether frontal patients and controls differed in terms of how specific the information needed to be in order for them to arrive at the correct response. That is, a participant could get 10 out of 10 trials correct but have made a disproportionately large number of guesses, arriving at the correct response only at the final sentence of the trial. In order to better understand the source of inferencing deficits on this test, we also calculated a number of other scores. These included measuring whether participants' 


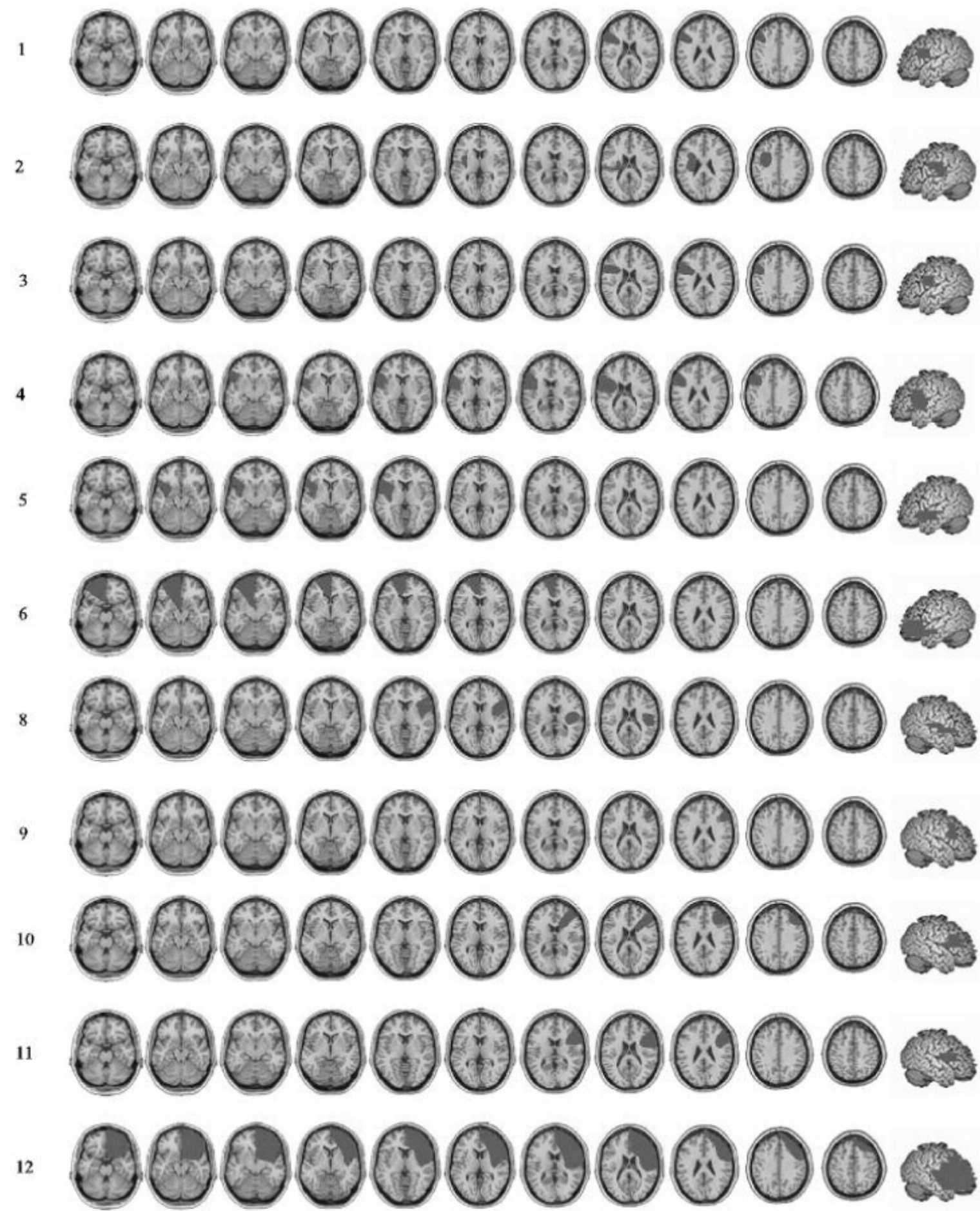

Fig. 1. Patients' lesions were reconstructed onto MRI templates. The far right image shows each patient's lesion projected onto the lateral surface of the brain. The numbers on the left side of the figure correspond to patients as listed in Table 1. Lesion reconstruction was unavailable for patient 7.

responses (1) made sense within the context of a given sentence; (2) made sense given the context of the preceding sentences of a trial; and (3) were consistent across sentences in a trial. These three scores overlap somewhat with the overall performance scores described above but were intended to further explore the source of inferencing deficits on this test.
The sentence context score was calculated to reflect how well a given response fit within the context of a particular sentence and thus whether a participant could integrate information within a single sentence. Responses were not scored on grammaticality but rather on how well they fit the sentence conceptually. The aim was to determine whether frontal patients could generate appropriate responses on a 
Table 2. Sample control and patient performance on the Word Context Test: Target item "Eat"

\begin{tabular}{lll}
\hline \hline Examiner & Control & Frontal \\
\hline Most people need to prifa several times a day. & Brush your teeth & Bathroom \\
Some people are very careful about what they prifa. & Eat & Say \\
You can prifa hot or cold things. & Eat & Cook \\
You need to $p r i f a$ to grow. & Eat & Cook \\
When you prifa, you put food in your mouth, chew, and swallow. & Eat & Use a fork \\
\hline \hline
\end{tabular}

sentence-by-sentence basis, even if they were unable to integrate that information across sentences. Three trained raters assigned a score of 1-3 to each response, based on the following criteria: $1=$ does not make sense, $2=$ makes some sense, and $3=$ makes very good sense. The total possible score per trial was 15 . The raters were blind as to the participants' identity, and the interrater reliability on this measure was .89 .

The integration score was calculated based on how well a given response fit the context provided by the preceding sentences. This score was computed in order to determine how well participants could integrate information across sentences, an ability we predicted would be impaired in frontal patients. Responses from only the $2 \mathrm{nd}$, 3rd, and 4th sentences of each trial were rated, since the first sentence did not have any preceding context, and the final sentence provided very obvious information as to the correct response. The same blind raters scored these responses, and the interrater reliability for the integration score was .82 .

The consistency score was calculated as the number of consecutively correct responses in the trial, divided by the number of the sentence (1-5) that first elicited the correct response. This ratio should be $100 \%$, if a participant maintains the correct response on subsequent sentences within a trial once s/he arrives at it. The lower this ratio is, the more indicative of set-loss problems. Thus, this score provided an indication of how well participants were able to maintain set, an ability often found to be affected in frontal patients.

In order to successfully integrate information on the Word Context Test, participants may need to maintain previous cue information, and possibly their strategy, in working memory for mental manipulation. Performance on the integration score was therefore compared to trial one of the CVLTII, to determine whether working memory played a role in inferential reasoning on this task. Data from the first trial of the CVLT-II were used in the current study as a measure of working memory, as this has been shown to be a reliable measure of verbal working memory and correlates with digit span (Delis et al., 1988).

Finally, as generation of verbal responses and word retrieval are processes integral to performance on the Word Context Test, the accuracy, total guesses, and sentence context measures were compared to semantic fluency, a measure of word retrieval ability (Baldo \& Shimamura, 1998).

\section{RESULTS}

A series of one-way analyses of variance were used to compare frontal patients' and controls' performance on the Word Context Test. With respect to overall performance, frontal patients' accuracy was impaired relative to controls $[F(1,22)=9.79, p<.01]$, as they were less able to infer the correct responses across the 10 trials $(M=7.83 / 10$, $S D=1.47$; and $M=9.33 / 10, S D=.78$, respectively). Frontal patients also performed worse than controls in that they required significantly more guesses to arrive at the correct response $[F(1,22)=9.40, p<.01 ; M=41.83 / 50$, $S D=8.31$; and $M=32.92 / 50, S D=5.70$, respectively $]$.

On the more qualitative measures, frontal patients also evidenced a number of weaknesses. Patients performed worse on the sentence context measure than controls $[F(1,22)=12.18, p<.01]$, as their responses were conceptually less appropriate given the context of each sentence $(M=13.42 / 15, S D=.99$; and $M=14.51 / 15, S D=$ .45 , respectively). We also compared how well patients and controls integrated information across sentences on a given trial. Again, frontal patients were impaired relative to controls $[F(1,22)=7.80, p<.05 ; M=2.28 / 3, S D=.45$; and $M=2.70 / 3, S D=.27$, respectively], suggesting that patients failed to adequately integrate information from the preceding sentences of a trial with the current sentence (see Table 2 for an example). On this measure, there were 2 participants ( 1 frontal and 1 control) whose responses were greater than 2 standard deviations below their group mean. After their data were removed from the analysis, the difference between frontal patients and controls was still significant $[F(1,20)=$ $11.66, p<.01]$.

Finally, the consistency score was calculated to reflect the number of consecutively correct responses within a trial and thus detect set-loss deficits. Patients and controls did not differ significantly on this measure $[F(1,22)=2.88$, $n s$; $M=88.21 / 100, S D=15.35$; and $M=96.14 / 100, S D=$ 5.20 , respectively], which would suggest that patients maintained set comparably to controls. However, this nonsignificant difference was likely due to the considerable degree of variability in the patients' performance on this measure.

We also examined the relationship between patients' performance on the Word Context Test and performance on two other measures, verbal fluency and Trial 1 of the CVLT-II. There was a significant correlation between ver- 
bal fluency and total number of guesses on the Word Context Test $(r=-.63, p<.01)$, as well as between verbal fluency and sentence context scores $(r=.65, p<.01)$ and accuracy scores $(r=.50, p<.05)$. There was no significant relationship, however, between the CVLT-II Trial 1 and integration scores $(r=.34, n s)$.

Although there were too few patients to statistically assess effects of side of lesion, descriptive analyses revealed that there was overlap in terms of performance between rightand left-frontal patients. Contrary to suggestions in previous research, patients with right-frontal lesions performed numerically better than patients with left-frontal lesions across a number of measures. Right-frontal patients were more accurate overall than patients with left-hemisphere lesions $(M=8.40, S D=1.34$ and $M=7.43, S D=1.51$, respectively) and required fewer guesses to arrive at the correct answer $(M=38.20, S D=9.58$ and $M=44.43$, $S D=6.83$, respectively). In addition, right-frontal patients received higher integration scores than those with leftfrontal lesions $(M=2.51, S D=.30$ and $M=2.12, S D=$ .48 , respectively). Effect size analyses revealed large differences between right and left frontal patients' performances with respect to accuracy (Cohen's $d=.68$ ), number of guesses (Cohen's $d=-.75)$, and integration scores (Cohen's $d=.97$ ). These estimates of effect size suggest that significant differences would be found between the groups if a large enough sample were available for parametric statistical analysis.

\section{DISCUSSION}

In the current study, patients with focal frontal lesions were impaired on a test of inferential reasoning that required them to determine the meaning of a made-up word based on its use in a series of sentences. Relative to a group of controls, frontal patients were less accurate on the Word Context Test and made more guesses overall. Planned analyses showed that this deficit was in part due to patients' inability to integrate information across sentences. It was also the case that frontal patients' responses were less appropriate within the context of a single sentence. Thus, frontal patients had difficulty inferring the meanings of unfamiliar words, both in the context of a single sentence as well as when integration across sentences was required.

In the current study, both left and right frontal patients performed worse than controls, however effect size analyses suggested that left frontal patients were worse than right frontal patients. This finding is not in keeping with literature suggesting a special role for the right frontal cortex and the right hemisphere generally in inferencing and language use (Brownell et al., 1983; Kaplan et al., 1990; Shammi \& Stuss, 1999; Siegal et al., 1996; Wapner et al., 1981). Our findings are in agreement with more recent findings that suggest that left-frontal regions are important for higher-level language tasks, such as inferencing and text comprehension (Channon \& Crawford, 2000; Ferstl et al.,
2002; Ferstl \& von Cramon, 2001). The current paradigm was quite different from previous studies of inferencing in that all of the stimulus material was present at the time of test, so that patients did not have to draw inferences across temporal lags. Nonetheless, frontal patients were impaired on the task.

The poor integration scores in frontal patients on the Word Context Test may reflect multiple functional deficits. It was apparent that patients with frontal lesions did not successfully update and integrate information. This finding is consistent with previous work that showed decreased integrative processing following prefrontal damage on a problemsolving task (Baldo et al., 2004). Previous studies have also shown that prefrontal damage is linked to an impaired ability to inhibit distracting information (Baldo \& Shimamura, 2000; Chao \& Knight, 1996) and suppress habitual responses (Perret, 1974). Thus, patients may have been unable to inhibit responding to the salience of certain words within a sentence, which would have led to a lack of integration with previous sentences.

Early research using a prior version of the Word Context Test with children suggested that errors on this task stemmed from cognitive rigidity, perseveration, and lack of verbal symbolism and abstractness (Werner \& Kaplan, 1950), deficits that have been associated with executive dysfunction (Keil \& Kaszniak, 2002). Similarly, recent studies have proposed that inferencing is negatively affected by cognitive inflexibility (Ferstl et al., 2002). Patients with left-frontal lesions have been shown to be more concrete in their responses compared to normal participants on language tasks (Novoa \& Ardila, 1987; Pearce et al., 1998), and such behaviors may have affected frontal patients' performance in the current study (see patient example, Table 2).

Although our study ruled out aphasic deficits in patients, the presence of subclinical linguistic deficits could have affected performance. Decreased performance on the Word Context Test was correlated with poor verbal fluency scores. This relationship suggests that impaired word retrieval may have affected patients' ability to generate appropriate words on the test. Ferstl et al. (2002) reported a similar relationship between decreased fluency and errors on an inferencing task, however, their task required a yes/no response. Thus, it is more likely that similar cognitive processes (e.g., strategic, non-automatic processes) underlie performance on both inferencing and verbal fluency tasks.

Such strategic processes, along with abstraction and integration, are commonly considered executive processes and likely play a critical role in performance on the Word Context Test. Not surprisingly, this patient group has been shown to suffer from a number of executive processing deficits (Baldo et al., 1998, 2002, 2004). The extent to which the Word Context Test measures cognitive processes distinct from executive dysfunction due to the frontal lesion cannot be determined through this study alone. Given the association between prefrontal cortex and working memory (D'Esposito et al., 1998; Goldman-Rakic, 1987; Smith \& Jonides, 1999), it is necessary to consider the possibility 
that frontal patients' impaired performance on the Word Context Test was simply a failure of working memory. There are two arguments against such an explanation. First, the sentence cues were always visible to participants, so previous cues did not need to be held in working memory. Second, there was no relationship between patients' scores on the Word Context Test and Trial 1 of the CVLT-II. Thus, it is unlikely that the patients' difficulties were due simply to insufficient working memory capacity. Consistent with these findings, another recent study also failed to find a relationship between working memory capacity and patients' ability to make inferences (Lehman-Blake \& Tompkins, 2001). Furthermore, previous work from our lab suggests that impaired working memory performance in this patient group stems in part from attentional and strategic deficits (e.g., Baldo \& Shimamura, 2000), rather than a frank loss of working memory capacity.

Generalizability of the current findings is limited by the small number of participants, which was due to the study's strict inclusionary criteria. Additionally, comparison to a neurologic control group may have provided evidence supporting a critical role for the prefrontal regions in successful inferencing, over and above non-frontal regions. Despite these limitations, however, we were able to show that deficits in inferencing can be observed even in non-aphasic patients with focal frontal lesions. These findings contribute to the growing body of literature recognizing subtle deficits in language processing that may occur in nonaphasic patients with frontal lobe lesions and suggest that inferential reasoning likely relies on an intact frontal system to support monitoring, updating, organizing, and integrating language.

\section{REFERENCES}

Alexander, M.P., Benson, D.F., \& Stuss, D.T. (1989). Frontal lobes and language. Brain and Language, 37, 656-691.

Baldo, J., Shimamura, A., Delis, D., Kramer, J., \& Knight, R. (1998). Performance of patients with frontal lobe lesions on a new executive function test battery. Abstract for the Cognitive Neuroscience Society.

Baldo, J.V., Delis, D., Kramer, J., \& Shimamura, A.P. (2002). Memory performance on the California Verbal Learning Test-II: Findings from patients with focal frontal lesions. Journal of the International Neuropsychological Society, 8, 539-546.

Baldo, J.V., Delis, D.C., Wilkins, D.P., \& Shimamura, A.P. (2004). Is it bigger than a breadbox? Performance of patients with prefrontal lesions on a new executive function test. Archives of Clinical Neuropsychology, 19, 407-419.

Baldo, J.V. \& Shimamura, A.P. (1998). Letter and category fluency in patients with frontal lobe lesions. Neuropsychology, 12, 259-267.

Baldo, J.V. \& Shimamura, A.P. (2000). Spatial and color working memory in patients with lateral prefrontal cortex lesions. Psychobiology, 28, 156-167.

Brownell, H.H., Michel, D., Powelson, J., \& Gardner, H. (1983). Surprise but not coherence: Sensitivity to verbal humor in righthemisphere patients. Brain and Language, 18, 20-27.
Brownell, H., Potter, H.H., \& Bihrle, A. (1986). Inference deficits in right brain-damaged patients. Brain and Language, 27, 310-321.

Channon, S. \& Crawford, S. (2000). The effects of anterior lesions on performance on a story comprehension test: Left anterior impairment on a theory of mind-type task. Neuropsychologia, 38, 1006-1017.

Chao, L. \& Knight, R. (1996). Human prefrontal lesions increase distractibility to irrelevant sensory inputs. NeuroReport, 6, 45-50.

Delis, D., Cullum, C.M., Butters, N., Cairns, P., \& Prifitera, A. (1988). Wechsler Memory Scale-Revised and California Verbal Learning Test: Convergence and divergence. Clinical Neuropsychologist, 2, 188-196.

Delis, D., Kaplan, E., \& Kramer, J. (2001). Delis-Kaplan Executive Function System. San Antonio, TX: The Psychological Corporation.

Delis, D., Kaplan, E., Kramer, J., \& Ober, B. (2000). California Verbal Learning Test-II. San Antonio, TX: The Psychological Corporation.

D’Esposito, M., Aguirre, G., Zarahn, E., Ballard, D., Shin, R., \& Lease, J. (1998). Functional MRI studies of spatial and nonspatial working memory. Cognitive Brain Research, 7, 1-13.

Ferstl, E.C., Guthke, T., \& von Cramon, D.Y. (2002). Text comprehension after brain injury: left prefrontal lesions affect inference processes. Neuropsychology, 16, 292-308.

Ferstl, E.C. \& von Cramon, D.Y. (2001). Change of perspective in discourse comprehension: Encoding and retrieval processes after brain injury. Brain and Language, 70, 385-420.

Freedman, M. \& Oscar-Berman, M. (1986). Bilateral frontal lobe disease and selective delayed response deficits in humans. Behavioral Neuroscience, 100, 337-342.

Goldman-Rakic, P. (1987). Circuitry of primate prefrontal cortex and regulation of behavior by representational memory. In F. Plum (Ed.), Handbook of physiology, nervous system, Vol. V: Higher functions of the brain, Part 1 (pp. 373-417). Bethesda: American Physiological Society.

Harden, W.D., Cannito, M.P., \& Dagenais, P.A. (1995). Inferential abilities of normal and right hemisphere damaged adults. Journal of Communication Disorders, 28, 247-259.

Henry, J.D. \& Crawford, J.R. (2004). A meta-analytic review of verbal fluency performance following focal cortical lesions. Neuropsychology, 18, 284-295.

Hier, D.B. \& Kaplan, J. (1980). Verbal comprehension deficits after right hemisphere damage. Applied Psycholinguistics, 1, 279-294.

Kaczmarek, B.L. (1984). Neurolinguistic analysis of verbal utterances in patients with focal lesions of frontal lobes. Brain and Language, 21, 52-58.

Kaplan, J.A., Brownell, H.H., Jacobs, J.R., \& Gardner, H. (1990). The effects of right hemisphere damage on the pragmatic interpretation of conversational remarks. Brain and Language, 38, 315-333.

Keil, K. \& Kaszniak, A.W. (2002). Examining executive function in individuals with brain injury: A review. Aphasiology, 16, 305-335.

Kertesz, A. (1982). Western Aphasia Battery. New York: Grune \& Stratton.

Lehman-Blake, M. \& Tompkins, C.A. (2001). Predictive inferencing in adults with right hemisphere brain damage. Journal of Speech, Language, and Hearing Research, 44, 639-654.

Lezak, M.D. (1995). Neuropsychological assessment (3rd ed.). New York: Oxford University Press. 
McDonald, S. (1993). Viewing the brain sideways? Frontal versus right hemisphere explanations of non-aphasic language disorders. Aphasiology, 7, 535-549.

McDonald, S. \& Pearce, S. (1996). Clinical insights into pragmatic theory: Frontal lobe deficits and sarcasm. Brain and Language, 53, 81-104.

Milner, B. \& Petrides, M. (1984). Behavioural effects of frontallobe lesions in man. Trends in Neuroscience, 7, 403-407.

Myers, P.S. \& Brookshire, R.H. (1996). Effect of visual and inferential variables on scene descriptions by right-hemispheredamaged and non-brain-damaged adults. Journal of Speech and Hearing Research, 39, 870-880.

Novoa, O.P. \& Ardila, A. (1987). Linguistic abilities in patients with prefrontal damage. Brain and Language, 30, 206-225.

Pearce, S., McDonald, S., \& Coltheart, M. (1998). Interpreting ambiguous advertisements: The effect of frontal lobe damage. Brain and Cognition, 38, 150-164.

Perret, E. (1974). The left frontal lobe of man and the suppression of habitual responses in verbal categorical behaviour. Neuropsychologia, 12, 323-330.
Shammi, P. \& Stuss, D.T. (1999) Humour appreciation: A role of the right frontal lobe. Brain, 122, 657-666.

Siegal, M., Carrington, J., \& Radel, M. (1996). Theory of mind and pragmatic understanding following right hemisphere damage. Brain and Language, 53, 40-50.

Smith, E.E. \& Jonides, J. (1999). Storage and executive processes in the frontal lobes. Science, 283, 1657-1661.

Troyer, A., Moscovitch, M., Winocur, G., Alexander, M., \& Stuss, D. (1998). Clustering and switching on verbal fluency. The effects of focal frontal- and temporal lobe lesions. Neuropsychologia, 36, 499-504.

Wapner, W., Hamby, S., \& Gardner, H. (1981). The role of the right hemisphere in the apprehension of complex linguistic materials. Brain and Language, 14, 15-33.

Werner, H. \& Kaplan, E. (1950). The acquisition of word meanings: A developmental study. Monographs of the Society for Research in Child Development, 15, Serial \#51. United Kingdom: Blackwell Publishing. 